\title{
CONTRAST SENSITIVITY OF THE CHANGING-SIZE CHANNEL
}

\author{
J. T. Petersik*, K. I. Beverley† and D. Regan $\dagger$ \\ *Department of Psychology, Southeast Missouri State University, Cape Girardeau, MO 63701, U,S.A. \\ and tDepartment of Physiology and Biophysics, Dalhousie University, Gerard Hall, \\ Halifax Infirmary, 5303 Morris St., Halifax, N.S. Canada B3J 1B6
}

(Received 18 February 1980; in revised form 29 October 1980)

\begin{abstract}
Two experiments investigated the contrast sensitivity of the visual changing-size channel, and in particular whether the perception of sideways motions and changing-size are mediated by different contrast-sensitive stages. In Experiment 1, subjects adjusted the contrast required to (1) detect, or (2) discriminate stimulus squares whose edges oscillated in either an antiphase or in an inphase manner. Oscillations varied in frequency $(0-8 \mathrm{~Hz})$ and in amplitude $\left(1^{\prime}-7^{\prime}\right.$ peak-to-peak). Contrast detection thresholds were significantly lower than discrimination thresholds, and both were generally independent of frequency. Thresholds for antiphase and inphase stimulation were not reliably different. The contrasts required to just see antiphase and inphase oscillations were elevated to the same extent after adaptation to an antiphase oscillation. In Experiment 2, subjects adjusted the oscillation amplitude required to detect stimulus oscillations after adaptation to antiphase stimulation $(2 \mathrm{~Hz})$ at various contrasts $(8.3 \%-86 \%)$. Elevations of the threshold oscillation amplitude saturated at about $50 \%$ adapting contrast, but were roughly proportional to log adapting contrast below $50 \%$. Antiphase threshold elevations were considerably greater than inphase elevations. The results are interpreted as supporting the notion that the threshold detection of both antiphase and inphase oscillating stimuli is mediated by a common early contrast-sensitive stage of analysis.
\end{abstract}

\section{INTRODUCTION}

The idea that the visual system acts as though it contained channels for changing-size is supported by psychophysical evidence (Regan and Beverley, 1978, 1980; Beverley and Regan, 1979), and is consistent with single neuron findings (Regan and Cynader, 1979; Regan et al., 1979a, b). In this article we describe the contrast sensitivity of the changing-size channel. In particular we discuss the question whether visual sensitivities to changing-size and sideways motion are mediated by different contrast-sensitive stages and whether they share a common contrast stage.

\section{METHODS}

We used the two forms of stimulation illustrated in Fig. 1A. Opposite edges of the square oscillated in opposite directions for antiphase stimulation, while opposite edges oscillated in the same direction for inphase stimulation. For antiphase oscillations the square's size oscillated around a mean value $\left(0.5^{\circ}\right)$ while the square remained stationary; the whole square oscillated in position along a diagonal during inphase oscillations. Each stimulus square initially subtended $0.5^{\circ}$ on any trial. In order to minimize errors caused by ocular tracking, two stimulus squares were arranged as illustrated in Fig. $1 B$ so that for inphase stimulation the two squares moved in opposite directions at any given time. Contrast was calculated as illustrated in Fig. 1C. The two stimulus squares were electronically generated on a Tektronix type 608 CRO with a green type 31 phosphor. The squares were optically superimposed on a $8^{\circ} \times 8^{\circ}$ green adapting background which was of luminance $11 \mathrm{~cd} / \mathrm{m}^{2}$. The dark fixation cross was in the centre of the background. The viewing distance was $145 \mathrm{~cm}$. Monocular viewing was used with the other eye occluded. Threshold data were gathered by the method of adjustment.

For Experiment 1 the subject adjusted a potentiometer which increased the intensity (and hence the contrast) of the stimulus squares. He reported when he first detected the presence of the squares. This setting was taken to be the detection threshold. He then continued to increase the luminance until he could discriminate whether the edges of the squares were oscillating inphase or antiphase. This setting was taken to be the discrimination threshold. The fact that the discrimination threshold was at a higher contrast than the detection threshold was an experimental finding. Frequent null trials when no squares were present, and static trials when no oscillations were present were included. Error rates were well below $1 \%$.

Six different stimulus oscillation frequencies of 0.25 , $0.5,1,2,4$ and $8 \mathrm{~Hz}$ were used. We randomized the oscillation frequency and whether the oscillations were inphase or antiphase. Four different stimulus oscillation amplitudes of 1, 2, 4 and $7 \mathrm{~min}$ arc peakto-peak per edge were used in separate experimental sessions on different days.

Rather than adjusting the contrast of the stimulus squares, in Experiment 2 the subject adjusted a poten- 
A
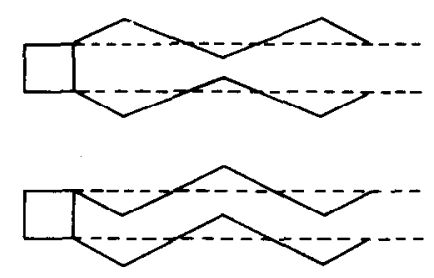

B
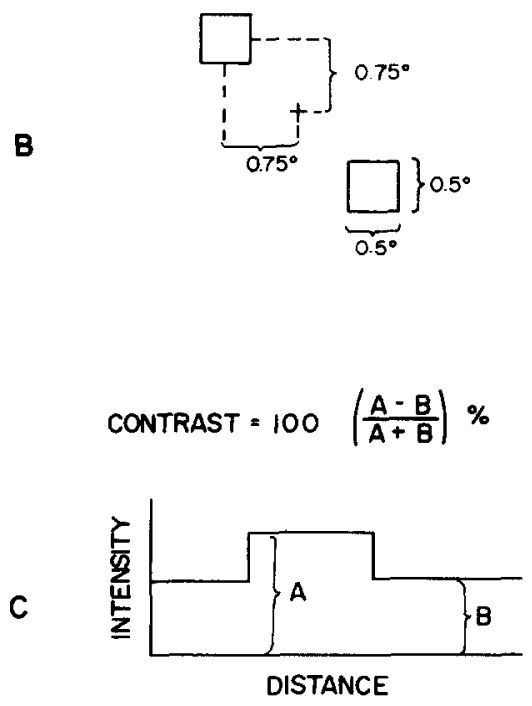

Fig. 1. Stimuli. (A) Shows the two stimulus oscillation conditions: antiphase or changing-size (upper) and inphase or changing-position (lower). Oscillations were $2 \mathrm{~Hz}$ triangular. The horizontal dimension shows the changes in the positions of stimulus edges over time. (B) Shows the stimulus configuration with two identical squares on either side of the fixation cross. (C) Definition of stimulus contrast.

tiometer which varied the oscillation amplitude of the edges of the stimulus squares. The setting at the point where he could just detect oscillations of one or both of the two squares was taken to be the threshold. Five test contrasts of $17,33,44,67$ and $86 \%$ were used. We randomized the test contrast and whether the oscillations were inphase or antiphase.

For $15 \mathrm{~min}$, subjects inspected an adapting stimulus of contrast $\mathrm{C} \%$ whose edges oscillated in antiphase at $2 \mathrm{~Hz}$ through $6 \mathrm{~min}$ arc peak-to-peak. Then the test stimulus was presented for $5 \mathrm{sec}$ during which the subject made a threshold setting. A 50-sec inspection of the adapting stimulus intervened between successive settings. Subjects made four settings at each of the 10 test stimuli in each experimental session. This experiment was carried out on separate days for each of the six different values of adapting contrast $\mathrm{C} \%$, namely $8.3,17,33,44,67$ and $86 \%$. "Baseline" measurements (stationary adapting stimulus) and

* Contrast thresholds to just detect the presence of the squares were very high. This was probably caused by (a) the eccentric fixation, (b) the small size of the stimuli and (c) the limited time allowed to make a setting. "adapted" measurements were gathered on separate days according to a random schedule. Threshold elevations were calculated as equal to 100 ("Adapted" - "Baseline")/"Baseline." This procedure was adopted because we wished to dissociate the effects of adapting to antiphase oscillations from the (considerable) effects of contrast adaptation.

Two experienced psychophysical subjects were used (the authors JTP and KIB).

\section{Experiment 1. Contrast required to detect inphase and antiphase oscillations}

Figure 2 shows the stimulus contrasts required for two separate tasks, namely (a) detecting the presence of the square (squares) and (b) discriminating whether the oscillations were inphase or antiphase (circles). Results are shown for both inphase (open symbols) and antiphase (filled symbols) test stimulus oscillations. Each panel shows the results for a different oscillation amplitude.

Figure 2 shows that the presence of the stimulus square could be detected at a considerably lower contrast than was required to detect that the square was oscillating rather than static $(15-40 \%$ for the largeamplitude oscillations; $10-15 \%$ for the small).* These differences were approximately the same for all frequencies tested $(0.25-8 \mathrm{~Hz})$. There was no consistent difference between the contrast required to detect inphase and antiphase oscillations. Thresholds were approximately independent of frequency from $1-8 \mathrm{~Hz}$, but rose below $1 \mathrm{~Hz}$. This rise may have been due, at least in part, to the increased difficulty at low frequencies of making a setting within $5 \mathrm{sec}$; at $0.25 \mathrm{~Hz}$ only 1.25 complete cycles occurred in $5 \mathrm{sec}$, whereas at $1 \mathrm{~Hz}$ and above the subject had the opportunity to integrate over five or more complete cycles. Thresholds were roughly similar for oscillation amplitudes of $7 \mathrm{~min}$ arc peak-to-peak per edge and $4 \mathrm{~min}$ arc peak-to-peak per edge, but considerably higher for the much weaker stimulus amplitude of $1 \mathrm{~min}$ arc peak-to-peak per edge.

Experiment 2. Threshold elevation produced by adaptation to antiphase stimuli of different contrasts

As mentioned earlier, in Experiment 2 subjects set thresholds by adjusting oscillation amplitude, while in Experiment 1 they set thresholds by adjusting stimulus contrast. We chose an oscillation frequency of $2 \mathrm{~Hz}$ in Experiment 2 since the data from Experiment 1 (Fig. 2) showed that thresholds were independent of frequency for frequencies near $2 \mathrm{~Hz}$.

After adapting to high-contrast square some of the low-contrast test targets could not be seen at all. For this reason, test contrasts below $40 \%$ could not be used with the $86 \%$ adapting contrast, and test contrasts below $20 \%$ could not be used with the $67 \%$ adapting contrast.

The plots of threshold elevation versus adapting contrast for the five test contrasts showed no consistent or large differences, so results for all five test con- 


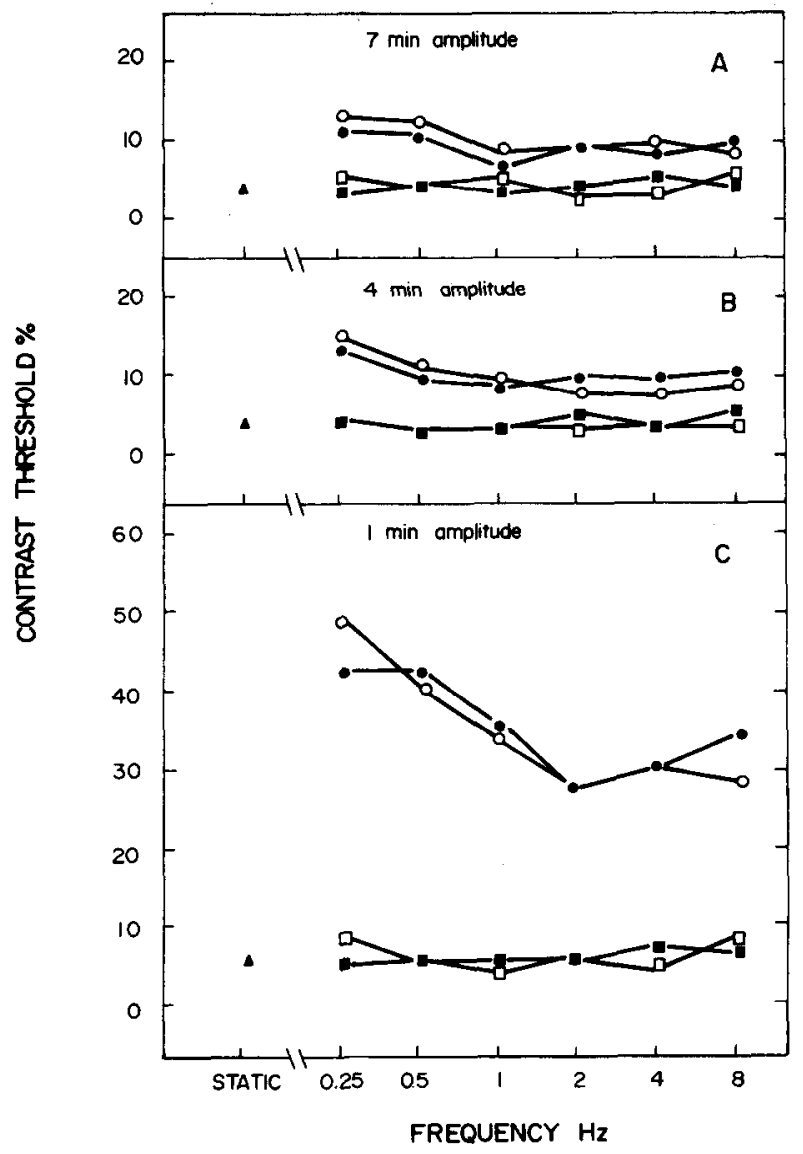

Fig. 2. Contrast thresholds for different oscillation frequencies. Ordinates plot the stimulus contrasts required to just detect the presence of the squares for antiphase oscillations (filled squares), inphase oscillations (open squares) or for static squares (filled triangles); or the stimulus contrasts required to just discriminate antiphase (filled circles) from inphase (open circles) stimulus oscillations. The amplitude of stimulus oscillation in min arc peak-to-peak was 7 in (A), 4 in (B) and 1 in (C). Each point is the mean of 4 settings. Subject KIB.

trasts were pooled together in Fig. 3 where, in each panel, threshold elevation is shown as a function of adapting contrasts for either antiphase test stimuli (filled symbols) or inphase test stimuli (open symbols). Panels A and B show results for subject JTP, C and D for KIB. Notice that for each panel of Fig. 3, the contrast required to just see the presence of a stimulus square is indicated by the leftmost arrow at $0 \%$ elevation (labeled "JSS"). Similarly, the contrast required to just see the oscillation of a stimulus square is indicated by the rightmost arrow at $0 \%$ elevation (labeled "JSO"). For subject JTP (Fig. 3A) threshold elevations for an antiphase test stimulus rose steeply at low adapting contrasts, and reached a plateau at an adapting contrast of about $40-50 \%$. A regression line was plotted through the data points for contrasts below and including $44 \%$. This line was extrapolated to zero threshold elevation. The intercept was somewhat above the contrast required to just see the stimulus square. This indicates that even though the stimulus square was visible, no threshold elevation was produced until a sufficiently high contrast was reached that stimulus oscillations could be seen, and thereafter threshold elevation was roughly proportional to $\log$ contrast until saturation occurred at about $50 \%$ adapting contrast.

As illustrated in Fig. 3B, adapting to an antiphase oscillation produced considerably smaller threshold elevations for inphase than for antiphase test stimuli. This asymmetry held for all five adapting contrasts used, and it is in accord with a previous report (Regan and Beverley, 1978).

Inphase threshold elevations saturated at about $50 \%$ adapting contrast in a similar manner to antiphase elevations, and when a regression line through the low-contrast points was extrapolated to zero threshold elevation, the intercept roughly coincided with the contrast required to just see stimulus oscillation. When the inphase threshold elevations of Fig. 3B were multiplied by a scaling factor of 2.25 , they agreed closely with the antiphase threshold elevations of Fig. 3A. The open circles in Fig. 3A are these inphase threshold elevations of Fig. $3 \mathrm{~B}$ multiplied by the scaling constant. 


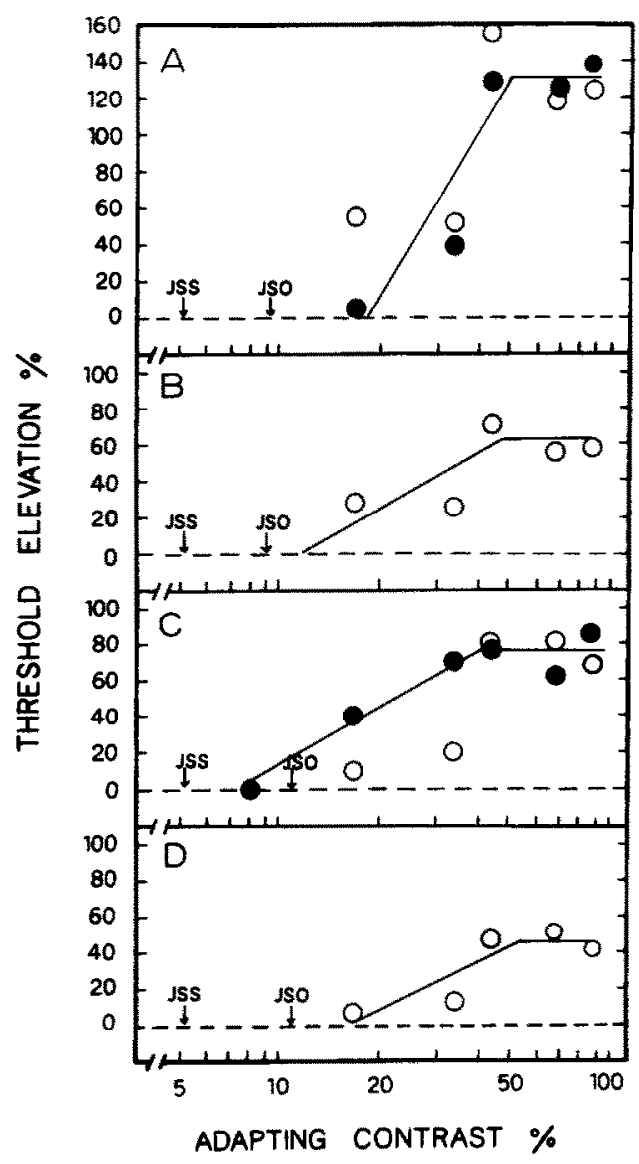

Fig. 3. Elevations of movement thresholds for different adapting contrasts. Threshold elevations (ordinate) were produced by inspecting an adapting square whose edges oscillated in antiphase through $6 \mathrm{~min}$ arc peak-to-peak at $2 \mathrm{~Hz}$. The contrast of the adapting square is plotted as abscissae. Results are pooled for test contrasts of 17, 33, 44, 67 and $86 \%$. (A) Test antiphase oscillations, subject JTP (filled circles). (B) Test inphase oscillations, subject JTP. (C) Test antiphase oscillations, subject KIB (filled circles). (D) Test inphase oscillations, subject KIB. JSS - Contrast required to just detect the presence of a stimulus square. ISO - contrast required to just detect oscillations of a stimulus square. Open circles in (A) and (C) are the data of (B) and (D) multiplied by the constant factors of 2.25 (subject JTP) or 1.66 (subject KIB). Each point is the mean of 40 setting for JTP and 20 settings for KIB.

Figure 3C shows that antiphase threshold elevations saturated at $40-50 \%$ adapting contrast for our second subject (KIB), although the "knee" in the curve was less marked than for subject JTP. For subject KIB a regression line was fitted to points below and including $44 \%$. For the inphase curve (Fig. 3D), two regression lines were not a better fit than a single line. However, when subject KIB's inphase threshold elevations were multiplied by a scaling factor of 1.66 , they approximated his antiphase threshold elevation.
We repeated Experiment 2 using as a threshold measure the contrast required to jest see a fixed oscillation amplitude of $6 \mathrm{~min}$ arc per edge peak-to-peak rather than the oscillation amplitude required to just see motion as in Fig. 3. Threshold elevations did not differ for inphase and antiphase stimuli.

The saturation of threshold elevations shown in Fig. 3 may be related to the saturation of patternreversal evoked potential amplitude at high contrasts (Spekreijse, 1966) and to the saturation of the classical motion aftereffect (Keck et al., 1976).

Our main conclusion is that there is a single contrast-sensitive stage in the visual analysis of movement, and that this stage is common to the changingsize channels and to the channels subserving threshold detection of inphase or side-to-side motion. Our evidence is as follows: (a) the contrasts required to just see inphase or antiphase oscillations were elevated to the same extent after adaptation to antiphase adapting stimuli; (b) the threshold elevations for inphase and antiphase test stimuli in Fig. 3 differed only by a scaling constant; (c) threshold elevations were independent of test contrast.

Acknowledgements-JTP's visit to Dahousie University was supported by a grant from the College of Education of Southeast Missouri State University. KIB was supported by the NRC of Canada (Grant A-0323). This research was sponsored by the Air Force Office of Scientific Research, Air Force Systems Command, USAF, AFOSR-78-3711. The United States Government is authorized to reproduce and distribute reprints for governmental purposes notwithstanding any copyright notation hereon.

\section{REFERENCES}

Beverley K. I. and Regan D. (1980) Visual Sensitivity to the shape and size of a moving object: Implications for models of object perception. Perception 9, 151-160.

Beverley K. I. and Regan D. (1979) Visual perception of changing size: the effect of object size. Vision Res. 19, $1093-1104$

Keck M. J., Palella T. D. and Pantle A. J. (1976) Motion aftereffect as a function of the contrast of sinusoidal gratings. Vision Res. 16, 187-191.

Regan D. and Beverley K. I. (1978) Looming detectors in the human visual pathway. Vision Res. 18, 415-421.

Regan D. and Beverley K. I. (1980) Independence of visual response to changing size and sideways motion for different directions of motion in depth. J. Opt. Soc. Am. October.

Regan D. and Beverley K. I. and Cynader M. (1979a) Stereoscopic sub-systems for position in depth and for motion in depth. Proc. R. Soc. Lond. B. 204, 485-501.

Regan D., Beverley K. I, and Cynader M. (1979b) The visual perception of motion in depth. Scient. Am. 241, 136-151.

Regan D. and Cynader M. (1979) Neurons in area 18 cat visual cortex selectively sensitive to changing size: nonlinear interactions between the response to two edges. Vision Res. 19, 688-711.

Spekreijse, J. (1966) Analysis of EEG response in man. Thesis. Junk, Amsterdam. 